Louisiana State University

LSU Digital Commons

Faculty Publications

Department of Chemistry

$10-28-2020$

\title{
Molecular motions of acetonitrile molecules in the solvation shell of lithium ions
}

Xiaobing Chen

Louisiana State University

Daniel G. Kuroda

Louisiana State University

Follow this and additional works at: https://digitalcommons.Isu.edu/chemistry_pubs

\section{Recommended Citation}

Chen, X., \& Kuroda, D. (2020). Molecular motions of acetonitrile molecules in the solvation shell of lithium ions. Journal of Chemical Physics, 153 (16) https://doi.org/10.1063/5.0024486

This Article is brought to you for free and open access by the Department of Chemistry at LSU Digital Commons. It has been accepted for inclusion in Faculty Publications by an authorized administrator of LSU Digital Commons.

For more information, please contact ir@lsu.edu. 


\section{Molecular motions of acetonitrile molecules in the solvation shell of lithium ions}

Cite as: J. Chem. Phys. 153, 164502 (2020); https://doi.org/10.1063/5.0024486

Submitted: 07 August 2020 - Accepted: 06 October 2020 • Published Online: 22 October 2020

Xiaobing Chen and (D) Daniel G. Kuroda

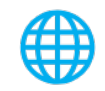

\section{ARTICLES YOU MAY BE INTERESTED IN}

Lithium-electrolyte solvation and reaction in the electrolyte of a lithium ion battery: A ReaxFF reactive force field study

The Journal of Chemical Physics 152, 184301 (2020); https://doi.org/10.1063/5.0003333

Effect of isotope substitution on the Fermi resonance and vibrational lifetime of unnatural amino acids modified with IR probe: A 2D-IR and pump-probe study of 4-azido-L-phenyl alanine

The Journal of Chemical Physics 153, 164309 (2020); https://doi.org/10.1063/5.0025289

Mixed quantum-classical treatment of electron transfer at electrocatalytic interfaces: Theoretical framework and conceptual analysis

The Journal of Chemical Physics 153, 164707 (2020); https://doi.org/10.1063/5.0009582

Challenge us.

What are your needs for periodic signal detection?

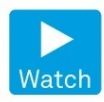

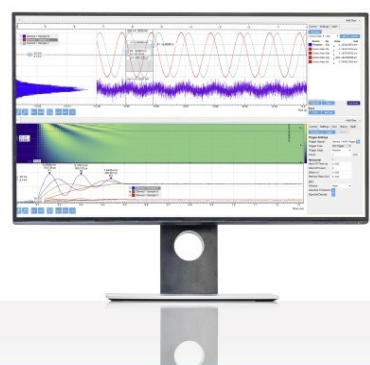

- Zurich Instruments 


\title{
Molecular motions of acetonitrile molecules in the solvation shell of lithium ions
}

\author{
Cite as: J. Chem. Phys. 153, 164502 (2020); doi: 10.1063/5.0024486 \\ Submitted: 7 August 2020 - Accepted: 6 October 2020 • \\ Published Online: 22 October 2020
}

Xiaobing Chen and Daniel G. Kuroda

\section{AFFILIATIONS}

Department of Chemistry, Louisiana State University, Baton Rouge, Louisiana 70803, USA

\author{
a) Author to whom correspondence should be addressed: dkuroda@lsu.edu
}

\begin{abstract}
Lithium ion solutions in organic solvents have become ubiquitous because of their use in energy storage technologies. The widespread use of lithium salts has prompted a large scientific interest in elucidating the molecular mechanisms, giving rise to their macroscopic properties. Due to the complexity of these molecular systems, only few studies have been able to unravel the molecular motions and underlying mechanisms of the lithium ion $\left(\mathrm{Li}^{+}\right)$solvation shell. Lately, the atomistic motions of these systems have become somewhat available via experiments using ultrafast laser spectroscopies, such as two-dimensional infrared spectroscopy. However, the molecular mechanism behind the experimentally observed dynamics is still unknown. To close this knowledge gap, this work investigated solutions of a highly dissociated salt [LiTFSI: lithium bis(trifluoromethanesulfonyl)imide] and a highly associated salt (LiSCN: lithium thiocyanate) in acetonitrile $(\mathrm{ACN})$ using both experimental and theoretical methods. Linear and non-linear infrared spectroscopies showed that $\mathrm{Li}^{+}$is found as free ions and contact ion pairs in ACN/LiTFSI and ACN/LiSCN systems, respectively. In addition, it was also observed from the non-linear spectroscopy experiments that the dynamics of the $\mathrm{ACN}$ molecules in the $\mathrm{Li}^{+}$first solvation shell has a characteristic time of $\sim 1.6$ ps irrespective of the ionic speciation of the cation. A similar characteristic time was deducted from ab initio molecular dynamics simulations and density functional theory computations. Moreover, the theoretical calculations showed that molecular mechanism is directly related to fluctuations in the angle between $\mathrm{Li}^{+}$and the coordinated $\mathrm{ACN}$ molecule $\left(\mathrm{Li}^{+} \cdots \mathrm{N} \equiv \mathrm{C}\right.$ ), while other structural changes such as the change in the distance between the cation and the solvent molecule $\left(\mathrm{Li}^{+} \cdots \mathrm{N}\right)$ play a minor role. Overall, this work uncovers the time scale of the solvent motions in the $\mathrm{Li}^{+}$solvation shell and the underlying molecular mechanisms via a combination of experimental and theoretical tools.
\end{abstract}

Published under license by AIP Publishing. https://doi.org/10.1063/5.0024486

\section{INTRODUCTION}

The solvation of lithium salts in organic solvents has gained significant attention in the last ten years due to its direct implication for designing electrolytes in the lithium ion battery (LIB). ${ }^{1-}$ In the past, most studies had focused on the macroscopic electrochemical aspects of the electrolytes, ${ }^{5-9}$ while only a few were able to study the molecular structure and dynamics of lithium ion electrolyte solutions. ${ }^{10-14}$ In particular, the problem of elucidating the molecular arrangements and interactions in lithium salt solutions arises from the ultrafast changes in the speciation of $\mathrm{Li}^{+}$and the fast motions of the molecular components. ${ }^{11-13,15-17}$ To this end, many experimental methodologies have been utilized to study the structure and dynamics of lithium ion electrolyte solutions, including Raman spectroscopy, ${ }^{18-20} \mathrm{x}$-ray scattering, ${ }^{21-23}$ nuclear magnetic resonance (NMR), ${ }^{24-26}$ mass spectrometry, ${ }^{27-29}$ and linear infrared spectroscopy. ${ }^{11,12,30-33}$ In addition, theoretical studies using different levels of theories have been presented. ${ }^{14,34-37}$ Notwithstanding the major findings of the previously used methodologies, the use of ultrafast infrared techniques to probe the molecular structure of lithium ion solutions has allowed us to gain dynamical information of the molecular motions occurring in these systems with a time resolution similar to that of the atomic. ${ }^{11,12}$

It is now well accepted that dilute solutions of lithium salts have the lithium ions $\left(\mathrm{Li}^{+}\right)$coordinating four solvent molecules (tetrahedral arrangement) when the solvent has one primary interaction site, such as carbonates and nitriles. ${ }^{11,14,36,38-40}$ At high concentration, the formation of contact ion pairs (CIPs), solvent separated ion pairs, and aggregates is observed, but the tetrahedral coordination of $\mathrm{Li}^{+}$is maintained. ${ }^{10,11,13,14,30,32,40}$ Time-resolved infrared 


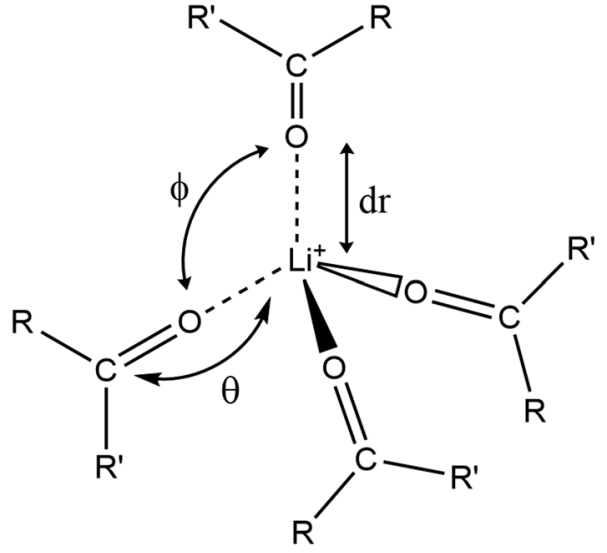

SCHEME 1. Geometrical factors describing the $\mathrm{Li}^{+}$solvation shell of carbonyl containing solvent molecules.

spectroscopy enables the investigation of the dynamics of the $\mathrm{Li}^{+}$ solvation shell. ${ }^{41}$ In particular, the motions of the first solvation shell have been probed through the carbonyl stretch of carbonate molecules coordinated to $\mathrm{Li}^{+}{ }^{11-13}$ From those studies, the dynamics of the $\mathrm{Li}^{+}$solvation shell was found to occur with a characteristic time of a few picoseconds. ${ }^{11,12}$ However, in the non-linear IR studies, it has been very difficult to decipher the molecular mechanisms giving rise to the observed dynamics due to the vibrational couplings that lead to a complex vibrational manifold for the solvent molecules in the $\mathrm{Li}^{+}$solvation shell.

In previous studies, the dynamics of the solvent (carbonates) molecules in the solvation shell were described in terms of three geometrical factors (Scheme 1): the distance between $\mathrm{Li}^{+}$and a carbonyl oxygen atom $\left(\mathrm{dr}, \mathrm{Li}^{+} \ldots \mathrm{O}\right)$, the angle between $\mathrm{Li}^{+}$and a coordinated carbonyl group $\left(\theta, \mathrm{Li}^{+} \ldots \mathrm{O}=\mathrm{C}\right)$, and the angle between two coordinated carbonyl groups $\left(\phi, \mathrm{O} \cdots \mathrm{Li}^{+} \cdots \mathrm{O}\right){ }^{11-13}$ It is found that the potential well for the distance between $\mathrm{Li}^{+}$and a coordinated carbonate molecule is narrow and deep, indicating that the thermal fluctuation of $\mathrm{dr}$ is highly restricted. ${ }^{12,14}$ While the distance between $\mathrm{Li}^{+}$and a single coordinated linear carbonate molecule appears to have an insignificant role in the $\mathrm{Li}^{+}$solvation shell motions, the fluctuations around the angles $\theta$ and $\phi$ emerge as the motions dominating the rearrangements of the solvation shell at room temperature. Subsequent theoretical work has further supported that the thermal changes in the angular factors dominate the motions of the $\mathrm{Li}^{+}$solvation shell since the characteristic times for the dynamics of their fluctuations match the dynamics observed in the experiments. ${ }^{11,14}$ It was also proposed that the most dominating factor in the motions of the $\mathrm{Li}^{+}$solvation shell is likely to be the angle $\phi$ as a result from the change in vibrational coupling for coordinated carbonyl stretches. Overall, the theoretical study supported the hypothesis that geometrical factors are good descriptors of the vibrational dynamics due to the strong vibrational coupling (excitonic nature) of the carbonyl stretch in carbonates. However, the excitonic nature of the vibrational manifold for coordinated carbonate molecules deterred the characterization of molecular motions of individual solvent molecules coordinated to $\mathrm{Li}^{+}$. Thus, the direct link between the experimentally observed dynamics and the specific molecular motions is still missing. For this purpose, a solvent presenting low vibrational coupling is needed to characterize the molecular motions of individual solvent molecules coordinated to $\mathrm{Li}^{+}$.

In this work, a combination of experimental and theoretical methods was employed to study the molecular mechanisms behind the dynamics of individual molecules in the $\mathrm{Li}^{+}$solvation shell. Acetonitrile (ACN-H3, Scheme 2) was chosen as a solvent because it has a dielectric constant higher than linear carbonates and, yet, comparable to cyclic carbonates. In addition, the ACN-H3 molecule coordinates exclusively to $\mathrm{Li}^{+}$through its nitrile group, which can be probed via its nitrile $(\mathrm{CN})$ stretch using IR spectroscopy, as previously demonstrated. ${ }^{38,42-44}$ To examine the CN stretching band, deuterated acetonitrile (ACN-D3) was used because it does not have the combination band found in the nitrile stretching region. ${ }^{45}$ Another key property of ACN is the transition dipole moment of its $\mathrm{CN}$ stretch, which is significantly lower than the corresponding carbonyl stretch of carbonates (see the supplementary material). Moreover, the small transition dipole of the nitrile stretch reduces substantially the vibrational coupling between adjacent molecules ${ }^{41}$ and permits the characterization of the motions of individual ACN molecules in the $\mathrm{Li}^{+}$solvation shell via time-resolved IR spectroscopy.

The motions of the $\mathrm{Li}^{+}$coordinated $\mathrm{ACN}$ molecules as a function of the ionic speciation of $\mathrm{Li}^{+}$were also investigated. For this purpose, two different salts were selected: lithium bis(trifluoromethanesulfonyl)imide (LiTFSI, Scheme 2) and lithium thiocyanate (LiSCN, Scheme 2). In the LiTFSI/ACN system, it has been previously shown that most cations have solvation shells formed exclusively by solvent molecules. ${ }^{46-49}$ In contrast, the ACN/LiSCN solutions have the $\mathrm{Li}^{+}$forming contact ion pairs. ${ }^{38,50-52}$ Thus, the structure and dynamics of ACN molecules in different $\mathrm{Li}^{+}$solvation shells were studied using linear and time-resolved infrared spectroscopies. In addition, the

$\mathrm{TFSI}^{-}$

$\mathrm{SCN}^{-}$

$\mathrm{Li}(\mathrm{ACN})_{4}^{+}$

$\mathrm{Li}(\mathrm{ACN})_{3} \mathrm{SCN}$
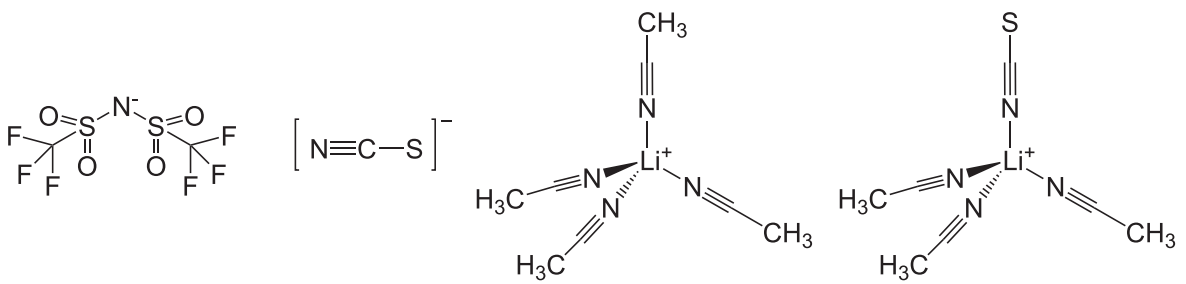

SCHEME 2. Chemical structure of the bis(trifluoromethanesulfonyl)imide ion $\left(\mathrm{TFSI}^{-}\right)$, thiocyanate ion $\left(\mathrm{SCN}^{-}\right)$, $\mathrm{Li}^{+}$coordinated to four acetonitrile molecules $\left[\mathrm{Li}(\mathrm{ACN})_{4}^{+}\right]$, and contact ion pair of $\mathrm{Li}^{+}$and $\mathrm{SCN}^{-}\left[\mathrm{Li}(\mathrm{ACN})_{3} \mathrm{SCN}\right]$. 
experimental studies were complemented with $a b$ initio computations and molecular dynamic simulations to uncover the molecular mechanism behind the motions of the ACN molecules coordinated to $\mathrm{Li}^{+}$.

\section{EXPERIMENTAL AND THEORETICAL METHODS}

\section{A. Sample preparation}

Tetrabutylammonium thiocyanate (TBASCN, 95\% TCI) was used as received. Lithium bis(trifluoromethanesulfonyl)imide (LiTFSI, 98\% Acros Organics), lithium thiocyanate hydrate ( $\mathrm{LiSCN} \cdot \mathrm{xH}_{2} \mathrm{O}, \mathrm{LiSCN}>63 \%$ Alfa Aesar), lithium perchlorate $\left(\mathrm{LiClO}_{4},>95 \%\right.$ Aldrich), acetonitrile (ACN-H3, 99\% Fisher Biotech), and acetonitrile-D3 (ACN-D3, 99.8\% isotope VWR) were dried before use. LiTFSI was dried in a vacuum oven under $150{ }^{\circ} \mathrm{C}$ for 2 days. $\mathrm{LiSCN} \cdot \mathrm{xH}_{2} \mathrm{O}$ was first dried in a vacuum oven under $60^{\circ} \mathrm{C}$ for 1 day and then under $110^{\circ} \mathrm{C}$ for 2 days. $\mathrm{LiClO}_{4}$ was dried in a vacuum oven under $140^{\circ} \mathrm{C}$ for 2 days. ACN-H3 and ACN-D3 were dried with molecular sieves for 2 days prior to use.

Solutions of either LiTFSI or LiSCN in ACN-D3 were prepared at different concentrations with $\mathrm{Li}^{+}$molar fractions of $0,0.01,0.025$, and 0.05 , where the molar fraction of $\mathrm{Li}^{+}$[i.e., $\mathrm{X}\left(\mathrm{Li}^{+}\right)$] is defined as the moles of $\mathrm{Li}^{+}$over the total moles of both $\mathrm{Li}^{+}$and solvent. Solutions of TBASCN and $\mathrm{LiClO}_{4}$ in $\mathrm{ACN}-\mathrm{H} 3$ were prepared at different $\mathrm{X}\left(\mathrm{Li}^{+}\right)$concentrations $(0,0.01,0.02,0.025$, and 0.05$)$, while the molar fraction of $\mathrm{SCN}^{-}$was kept at $\mathrm{X}\left(\mathrm{SCN}^{-}\right)=0.05$ in all solutions. Note that TBASCN was used to describe the free ion because it has been previously observed that its presence does not perturb the anion, which allowed us to investigate the speciation of the thiocyanate ion. Sample cells for Fourier transform infrared (FTIR) spectroscopy and 2DIR consisted of the ACN-D3/ $\mathrm{Li}^{+}$solution sandwiched between two $\mathrm{CaF}_{2}$ windows with or without spacer according to the required optical density of the sample. All samples and sample cells were prepared in a $\mathrm{N}_{2}$-filled glovebox to minimize water contamination. All prepared samples tested less than $100 \mathrm{ppm}$ of water after preparation. Note that in the rest of this article, ACN refers to ACN-D3 unless otherwise noted.

\section{B. Fourier transform infrared spectroscopy (FTIR)}

FTIR experiments were performed on a Bruker Tensor 27 equipped with a liquid nitrogen cooled MCT detector with a spectral resolution of $0.5 \mathrm{~cm}^{-1}$. Reported spectra were averaged over 40 scans. FTIR data were modeled using OriginLab software.

\section{Two-dimensional (2D) IR spectroscopy}

The setup used for 2DIR experiments has been previously detailed in the literature, so only a short description is provided here. ${ }^{41,53}$ The input IR pulses were generated with a Spectra Physics Spitfire Ace Ti:sapphire amplifier at a repetition rate of $5 \mathrm{kHz}$, in combination with an OPA-800C and difference frequency generation crystal. These input IR pulses were then split into three replicas and later focused on the sample using the well-known boxcars geometry. ${ }^{54}$ The photon echo signal was measured in the $-\mathrm{k}_{1}+\mathrm{k}_{2}+\mathrm{k}_{3}$ phase-matching direction. A heterodyned detection was performed using a fourth pulse (local oscillator). The heterodyned signal was measured in a 64-element MCT array detector after dispersing the heterodyned signal in a spectrometer. The photon echo signal was measured as a function of three critical time intervals: the coherence time $\tau$ (interval between pulses 1 and 2 ), the waiting time $T_{\mathrm{w}}$ (interval between pulses 2 and 3 ), and the coherence time $t$ (interval between pulse 3 and the detected signal). These time intervals were set via computer-controlled translation stages. Here, 2DIR data were collected by scanning $\tau$ time from -4 ps to +4 ps in increments of 5 fs for each waiting time to collect both the rephasing and nonrephasing data by switching the time ordering. ${ }^{55}$ Signals were collected for waiting times from 0 ps to 3 ps in steps of 0.25 ps. The data collection in waiting time was confined to a maximum of 3 ps due to the presence of heating effects. ${ }^{32,56,57}$ In all the measurements, the local oscillator always preceded the photon echo signal by $\sim 0.6 \mathrm{ps}$. The time domain signal, collected as a function of $\left(\tau, T_{w}, \lambda_{t}\right)$ via a monochromator-array detection, is transformed into the 2DIR spectra $\left(\omega_{\tau}, T_{w}, \omega_{t}\right)$ by means of Fourier transforms. A detailed explanation of the Fourier analysis has been described elsewhere.

\section{D. $\boldsymbol{A b}$ initio molecular dynamics simulations (AIMDS)}

The $a b$ initio molecular dynamics simulations (AIMDSs) were carried out with the CP2K package (version 3.0). ${ }^{59}$ The electronic structure was calculated via a Quickstep module ${ }^{59}$ using the PBE functional with the $\mathrm{D} 2^{60}$ dispersion scheme and the TZV2P basis set, with Goedecker-Teter-Hutter (GTH) pseudopotentials. ${ }^{61-63}$ Periodic boundary conditions were applied to all the systems, and the Nosé-Hoover thermostat was used to keep the temperature constant at $300 \mathrm{~K}$, with the temperature damping constant of 100 fs. A self-consistent field (SCF) convergence criterion was set to $5.0 \times 10^{-7}$ hartree. The plane wave cutoff was set to 400 Ry. ${ }^{64}$ The ACN/LiTFSI system was composed of one lithium ion $\left(\mathrm{Li}^{+}\right)$, one bis(trifluoromethanesulfonyl)imide ion (TFSI ${ }^{-}$), and 61 acetonitrile-t3 (ACN-T3) in a cubic box with a length of $\sim 17.5 \AA$. ACN-T3 [i.e., all hydrogens $(\mathrm{H})$ were replaced by tritium $(\mathrm{T})$ ] is required for $1.0 \mathrm{fs}$ time steps in the AIMDSs. ${ }^{35,65}$ Similarly, the ACN/LiSCN system was composed of one $\mathrm{Li}^{+}$, one thiocyanate ion, and $61 \mathrm{ACN}-\mathrm{T} 3$ molecules in a cubic box with a length of $\sim 17.5 \AA$ A. In this paper, both box compositions are representative of dilute lithium solutions in acetonitrile [i.e., $\mathrm{X}\left(\mathrm{Li}^{+}\right) \leq 0.05$ ] since aggregates are not found at significant concentrations in dilute solutions. $^{10,13,44,66}$

The initial lithium solvation shell of LiTFSI in ACN-T3, consisting of a $\mathrm{Li}^{+}$coordinated to four acetonitrile molecules (tetrahedral solvation shell), was first optimized by Gaussian $09 .{ }^{67}$ Similarly, the initial structure of the LiSCN contact ion pair in ACN-T3, consisting of a $\mathrm{Li}^{+}$coordinated to three acetonitrile molecules and one $\mathrm{SCN}^{-}$, was also optimized by Gaussian $09 .{ }^{67}$ Each optimized solvation shell was then submerged in a box containing the rest of the acetonitrile molecules using PACKMOL. ${ }^{68}$ The molecular box with restraints on the molecules forming the first solvation shell of lithium underwent a minimization run over 500 cycles, a 2 ns NVT, and 2 ns NPT process using an AMBER software package and GAFF force field. ${ }^{69,70}$ These preparation steps were required to assure the equilibration of the box while maintaining the correct solvation structure around $\mathrm{Li}^{+}$. In the starting structure of AIMDS, the distance between $\mathrm{Li}^{+}$and the $\mathrm{N}$ atom of TFSI ${ }^{-}$was set at $9.6 \AA$ in the ACN/LiTFSI system, while the distance between $\mathrm{Li}^{+}$and the 


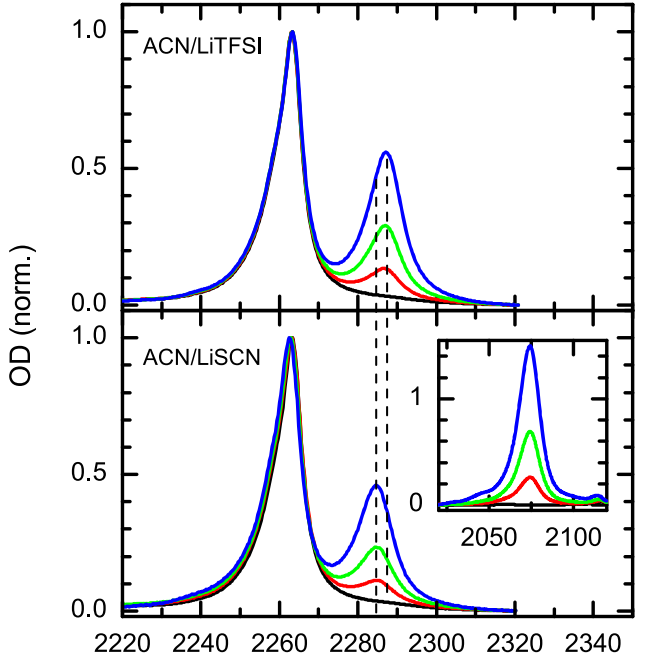

FIG. 1. Concentration-dependent FTIR spectra in the nitrile stretch region. Top panel corresponds to ACN/LiTFSI in the ACN nitrile stretch region, and the bottom panel corresponds to $A C N / L i S C N$ in the $A C N$ nitrile stretch region. The inset in the bottom panel shows the same sample in the thiocyanate nitrile stretch region. The display concentrations are $X\left(\mathrm{Li}^{+}\right)=0$ (black), $X\left(\mathrm{Li}^{+}\right)=0.01($ red $), X\left(\mathrm{Li}^{+}\right)=0.025$ (green), and $X\left(\mathrm{Li}^{+}\right)=0.05$ (cyan).

$\mathrm{N}$ atom of the thiocyanate ion was set at $2.1 \AA$ in the ACN/LiSCN system. In the AIMDSs, the systems were equilibrated for $\sim 20 \mathrm{ps,}$ and the production run was carried out in the NVT ensemble for another $\sim 180$ ps.

\section{E. DFT calculations}

Density functional theory (DFT) calculations were performed with Gaussian 09 software at the PBE1PBE level of theory using the $6-311++\mathrm{G}^{* *}$ basis set. ${ }^{67}$ In order to obtain reasonable results of structure optimizations and frequency calculations, the initial geometries of the lithium solvation shells were captured from the AIMDS. The captured structure of the tetrahedral solvation shell and contact ion pair contained not only the first solvation shells of $\mathrm{Li}^{+}$ but also an appropriate amount of acetonitrile molecules from the second solvation shell. Structure optimization and frequency calculations were performed in the gas phase unless otherwise noted.

\section{RESULTS}

\section{A. FTIR spectroscopy}

Linear IR spectroscopy was first used to study the structure of LiTFSI and LiSCN in ACN. Concentration-dependent infrared (IR) spectra in the CN stretching region of ACN $\left(2220 \mathrm{~cm}^{-1}-2320 \mathrm{~cm}^{-1}\right)$ are shown in Fig. 1. In pure ACN, only one asymmetric band is observed at $\sim 2260 \mathrm{~cm}^{-1}$. This band is asymmetric due to an overlap of hot band transition in the low-frequency side. ${ }^{71-73}$ The addition of the lithium salt gives rise to a new band at the high-frequency side $\left(\sim 2285 \mathrm{~cm}^{-1}\right)$ of the solvent CN stretch band, which becomes more pronounced when the concentration of $\mathrm{Li}^{+}$increases. Due to the direct increase in the high frequency band with the addition of salt, the low-frequency and high-frequency bands have been assigned to free and $\mathrm{Li}^{+}$-coordinated $\mathrm{CN}$ stretching bands, respectively. ${ }^{38}$ It is to be observed that the spectra reported here are similar to the spectra from previous studies on similar systems. $38,42-44,74,75$ In addition, it is observed in the case of LiSCN in ACN that a band at $2074 \mathrm{~cm}^{-1}$ grows with the addition of the lithium salt. This downshifted band corresponds to the nitrile stretch of the anion.

It is now well accepted that at low concentrations, $\mathrm{Li}^{+}$and $\mathrm{TFSI}^{-}$remain dissociated in high dielectric organic solvents, such as ACN. ${ }^{46-49}$ In addition, previous research has evidenced a solvent coordination number of 4 in dilute $\mathrm{ACN} / \mathrm{Li}^{+}$solutions. ${ }^{38,42-44,47,74,75}$ (a)

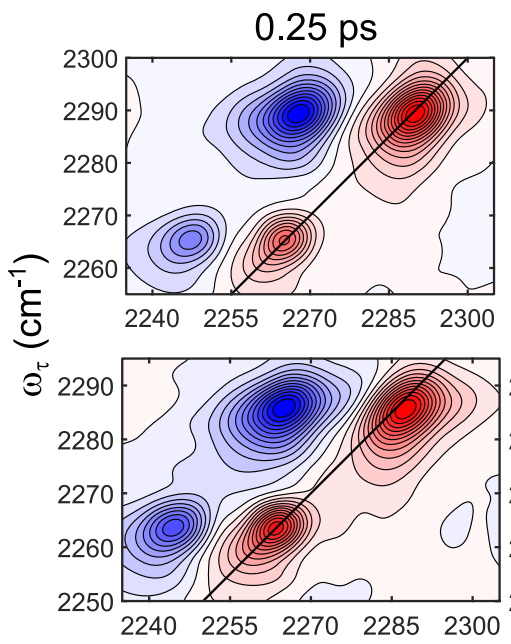

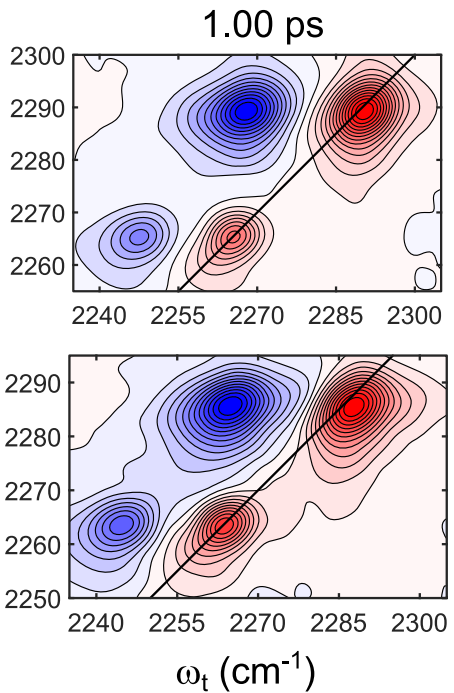

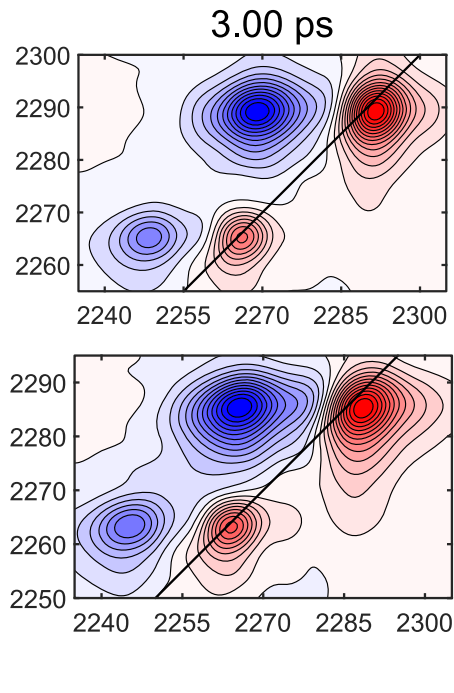

FIG. 2. 2DIR spectra of (a) LiTFSI in ACN (top panels) and (b) LiSCN in ACN (bottom panels) with X(Li+) equal to 0.05 . The spectra at the waiting times of $0.25 \mathrm{ps}, 1 \mathrm{ps}$, and $3 \mathrm{ps}$ are shown in left, middle, and right panels, respectively. 2DIR spectra at waiting time 0 are shown in the supplementary material. 
Thus, it is expected that most $\mathrm{Li}^{+}$will coordinate four ACN molecules at the investigated concentrations $\left[0 \leq \mathrm{X}\left(\mathrm{Li}^{+}\right) \leq 0.05\right]$ in the ACN/LiTFSI system. On the other hand, LiSCN has been proven to be a highly associated salt that can form contact ion pairs (CIPs) in organic solvents. ${ }^{38,50-52}$ The speciation of $\mathrm{SCN}^{-}$, characterized using its $\mathrm{CN}$ stretch band allows us to reach the same conclusion. First, a sample containing $\mathrm{SCN}^{-}$with a non-perturbing cation (TBA) shows a peak at $2057 \mathrm{~cm}^{-1}$ (see the supplementary material), which is downshifted by $\sim 20 \mathrm{~cm}^{-1}$ from the peak seen for LiSCN in ACN (Fig. 1). Second, a concentration-dependence of a mixture of TBASCN with varying amounts of $\mathrm{LiClO}_{4}$ shows the rise of the CIP peak at $\sim 2075 \mathrm{~cm}^{-1}$ with the addition of $\mathrm{Li}^{+}$(see the supplementary material). These results are similar to observations previously made for LiSCN in dimethylformamide, which is a high dielectric constant solvent. $^{5}$

\section{B. 2DIR spectroscopy}

To gain more structural and dynamical information of the $\mathrm{ACN} / \mathrm{Li}^{+}$solutions, the 2DIR spectra were collected for the two systems ACN/LiTFSI and ACN/LiSCN at $\mathrm{X}\left(\mathrm{Li}^{+}\right)=0.05$. The 2DIR spectra in the region corresponding to the $\mathrm{CN}$ stretch of $\mathrm{ACN}$ are shown in Fig. 2. The data were analyzed for waiting times up to 3 ps due to the presence of heating effects. ${ }^{32,50,56,76}$ In both solutions, the 2DIR spectra have two pairs of peaks on top of the diagonal line represented by the black solid line. These red positive peaks are attributed to the third order non-linear response arising from the transitions between the $v=0$ and $v=1$ vibrational states of ACN, while the blue negative peaks downshifted by $\sim 24 \mathrm{~cm}^{-1}$ correspond to non-linear signals arising from the transitions between the $v=1$ and $v=2$ vibrational states. The peaks on the low-frequency and high-frequency sides of the 2DIR spectra have direct correspondence to the free and coordinated CN bands seen in the FTIR spectra, respectively.

The waiting time evolution of the spectra shows that the main peaks are more tilted along the diagonal line at early waiting time, indicating that the pump and probe frequencies are correlated. However, the 2DIR peaks become more upright at later waiting times, depicting the loss of correlation between the pump and probe frequencies as the waiting time progresses. The peak shape dependence with waiting time shows the randomization of the initially pumped frequencies (spectral diffusion process) in these systems. ${ }^{41}$ Interestingly, the peak shapes evolve rapidly within the first 2 ps and only change marginally afterward for both samples. In addition, the 2DIR spectra do not present off-diagonal features within the first 2 ps.

\section{DISCUSSION}

\section{A. Speciation of $\mathrm{Li}^{+}$}

The FTIR spectra (Fig. 1) show only two peaks in the CN stretching region of ACN for either LiTFSI or LiSCN samples. The result indicates that in each solution, the solvent is mainly observing two different molecular environments, i.e., the bulk solvent and $\mathrm{Li}^{+}$ solvation shell. $\mathrm{Li}^{+}$is known to form a tetrahedral solvation shell in most organic solvents with single coordination sites. ${ }^{11,47,77-80}$ Thus, the coordinated peak (high frequency) seen in the ACN/LiTFSI samples corresponds to the ACN molecules in the first solvation shell of $\mathrm{Li}^{+}$. Moreover, the coordinated peak positions relative to the free peaks and the full width at half maximum (FWHM) of the coordinated peak (Fig. 3) remain almost invariant with an increase in $\mathrm{Li}^{+}$concentration, demonstrating that there is no obvious change in the speciation of $\mathrm{Li}^{+}$within the investigated range of concentrations. This last result is in agreement with the large dissociation presented by this salt in $\mathrm{ACN} .{ }^{47}$ In the case of $\mathrm{ACN} / \mathrm{LiSCN}, \mathrm{Li}^{+}$predominantly exists as a contact ion pair with a tetrahedral solvation shell, which includes $\mathrm{SCN}^{-}$. It is important to note that in the LiSCN CIP, the anion coordinates $\mathrm{Li}^{+}$through the $\mathrm{N}$ atom rather than the $\mathrm{S}$ atom (see the supplementary material). ${ }^{50}$ Thus, the two peaks at the lowand high-frequency sides seen in the $\mathrm{CN}$ stretching region of $\mathrm{ACN}$ for LiSCN are assigned to free and $\mathrm{Li}^{+}$-coordinated $\mathrm{ACN}$ molecules in the CIP, respectively. Similar to the case of LiTFSI, the spectra of ACN/LiSCN show a constant relative peak position and FWHM as functions of $\mathrm{Li}^{+}$concentrations (Fig. 3).

The FTIR spectra of ACN/LiTFSI and ACN/LiSCN appear to be similar (Fig. 1), but a close inspection reveals that the relative peak position of the coordinated $\mathrm{CN}$ of $\mathrm{ACN}$ is slightly more upshifted for LiTFSI samples $\left(\sim 25 \mathrm{~cm}^{-1}\right)$ as compared to LiSCN samples $\left(\sim 23 \mathrm{~cm}^{-1}\right)$. This difference in the relative peak position is attributed to the different speciation of $\mathrm{Li}^{+}$, which in the case of LiTFSI is mainly $\mathrm{Li}(\mathrm{ACN})_{4}^{+}$and in the case of $\mathrm{LiSCN}$ is $\operatorname{Li}(\mathrm{ACN})_{3}(\mathrm{SCN})$. The assignment is supported by DFT computations, where a similar upshift of the CN stretch of $\mathrm{ACN}$ is seen for the $\mathrm{Li}(\mathrm{ACN})_{4}{ }^{+}$ when compared to $\mathrm{Li}(\mathrm{ACN})_{3} \mathrm{SCN}$ solvation shells (Fig. 4). Finally, the deduced speciation is also in agreement with the AIMDSs. The radial distribution functions (RDFs) between $\mathrm{Li}^{+}$and the nitrogen atom of the coordinated $\mathrm{ACN}\left[\mathrm{g}(\mathrm{r}) \mathrm{Li}-\mathrm{N}_{\mathrm{ACN}}\right]$ and the integral of $g(r) L i-N_{A C N}\left[\operatorname{int}\left(g(r) L i-N_{A C N}\right)\right]$ show that in the LiTFSI system, $\mathrm{Li}^{+}$coordinates four ACN molecules, but it coordinates three ACN molecules and one thiocyanate ion in the LiSCN system (Fig. 4). A similar molecular picture is deduced for the LiSCN/ACN system

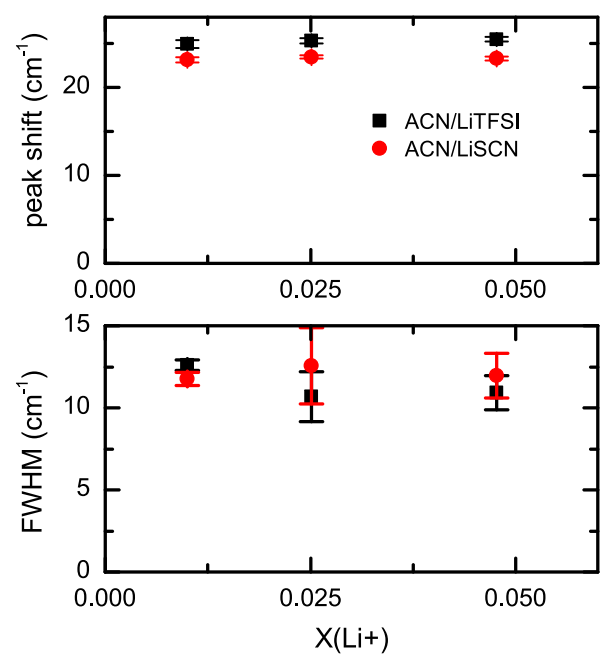

FIG. 3. Line shape parameters of the coordinated nitrile stretch of ACN as a function of $\mathrm{Li}^{+}$concentration. Top and bottom panels display the peak shift from free peaks to coordinated peaks and the full width at half maximum (FWHM) of coordinated peaks, respectively. Black squares and red circles represent the ACN/LiTFS and ACN/LiSCN samples, respectively. 

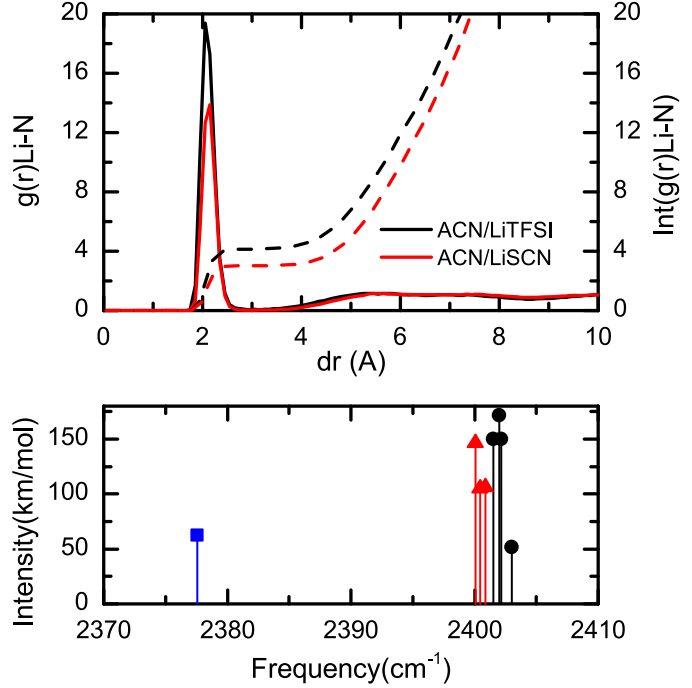

FIG. 4. Computational results. The top panel shows the radial distribution function (RDF) between $\mathrm{Li}^{+}$and the nitrogen atom of the coordinated ACNs $\left[\mathrm{g}(\mathrm{r}) \mathrm{Li}^{+}-\mathrm{N}_{\mathrm{ACN}}\right.$, solid line] and its integrated RDF [int $\left(\mathrm{g}(\mathrm{r}) \mathrm{Li}^{+}-\mathrm{N}_{\mathrm{ACN}}\right)$, dashed line] from AIMDS. Black and red correspond to the LiTFSI and LiSCN solutions in ACN, respectively. The bottom panel depicts the DFT frequency calculations (PCM) for free and coordinated ACN. Blue squares, black circles, and red triangles represent the frequencies and intensities of the nitrile stretch in a single ACN molecule, in a tetrahedral solvation shell of free $\mathrm{Li}^{+}\left[\mathrm{Li}(\mathrm{ACN})_{4}{ }^{+}\right]$, and its $\mathrm{CIP}\left[\mathrm{Li}(\mathrm{ACN})_{3}(\mathrm{SCN})\right]$, respectively. The geometry used for these calculations can be found in the supplementary material.

from the radial distribution function between $\mathrm{Li}^{+}$and the nitrogen atom of thiocyanate (see the supplementary material).

\section{B. Vibrational coupling}

The experiments performed here were designed with the objective of characterizing the molecular motions of the individual solvent molecules coordinating $\mathrm{Li}^{+}$. Previous attempts on other solvents did not succeed because of the strong coupling among solvent molecules in the $\mathrm{Li}^{+}$solvation shell. ${ }^{11-13}$ In contrast with previous systems, ACN has a small transition dipole magnitude, which is $\sim 20$ times smaller than dimethyl carbonate (see the supplementary material). The small transition dipole for the $\mathrm{CN}$ stretch of ACN is directly observed when computing the vibrational spectrum of the complex $\operatorname{Li}(\mathrm{ACN})_{4}{ }^{+}$. In this case, the separation between the symmetric and asymmetric $\mathrm{CN}$ stretches is only $\sim 2 \mathrm{~cm}^{-1}$ (Fig. 4 ), demonstrating that the vibrational coupling constant is small $\left(\beta<0.5 \mathrm{~cm}^{-1}\right)$. Experimentally, this is confirmed by the FTIR and 2DIR spectra of the ACN/LiTFSI solution (Fig. 2) since only one peak is observed in the coordinated region in agreement with the symmetric and asymmetric $\mathrm{CN}$ stretches being very close to one another in frequency. In addition, the cross peaks due to vibrational coupling between the coordinated and free ACN molecules are absent at all measured waiting times.

The lack of cross peaks between the coordinated and free peaks is in stark contrast with the observation in solutions of solvents containing carbonyl groups. ${ }^{11-13,16,31}$ For example, the 2DIR spectra of solution of $\mathrm{Li}^{+}$in organic carbonates and ureas presented cross peaks between the asymmetric and symmetric carbonyl stretch modes in 2DIR spectra at $\mathrm{T}_{\mathrm{w}}=0 \mathrm{ps}$. Moreover, the characteristic time of vibrational energy transfer in the carbonate $/ \mathrm{Li}^{+}$or urea $/ \mathrm{Li}^{+}$ system is found to be on the order of several to tens of picosecond. ${ }^{11-13,31}$ However, in the case of $\mathrm{ACN} / \mathrm{Li}^{+}$solutions, the cross peaks are not observed within the investigated window of $\mathrm{T}_{\mathrm{w}}=3 \mathrm{ps}$. The lack of cross peaks between the coordinated and free peaks also demonstrates that the chemical exchange characteristic time is much slower than a few picoseconds, but it does not imply that the $\mathrm{ACN}$ molecules in the $\mathrm{Li}(\mathrm{ACN})_{4}{ }^{+}$complex are not vibrationally coupled. On the contrary, the small vibrational coupling among ACN molecules makes the cross peak due to vibrational coupling between the symmetric and asymmetric $\mathrm{CN}$ stretches to be located within the diagonal peak of the $\mathrm{CN}$ stretches of coordinated $\mathrm{ACN}$ molecules.

The weak vibrational coupling is also seen by computing distortions of the $\mathrm{Li}^{+}$solvation shell. In this case, adjusting the distance $\left(\mathrm{dr}, \mathrm{N} \cdots \mathrm{Li}^{+}\right)$or the angles $\theta\left(\mathrm{C} \equiv \mathrm{N} \cdots \mathrm{Li}^{+}\right)$and $\phi\left(\mathrm{N} \cdots \mathrm{Li}^{+} \cdots \mathrm{N}\right)$ of one $\mathrm{ACN}$ within the $\mathrm{Li}(\mathrm{ACN})_{4}{ }^{+}$complex does not significantly affect the $\mathrm{CN}$ frequencies of the other coordinated molecules (Fig. 5). This result further supports our hypothesis that the coupling between coordinated CN groups is weak.

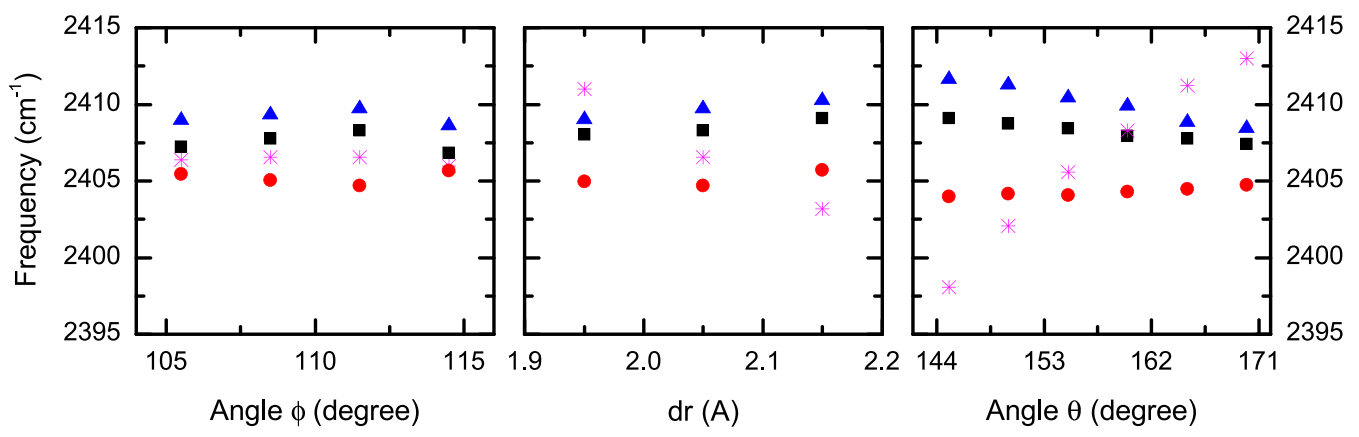

FIG. 5. Frequency changes in the nitrile stretches computed by DFT as a function of different geometrical factors: angle $\phi$ (left), distance dr (middle), and angle $\theta$ (right) in a solvation shell of $\mathrm{Li}(\mathrm{ACN})_{4}{ }^{+}$. Magenta star, black square, red circle, and blue triangle represent the calculated frequencies of the four coordinated nitriles of ACN. Note that only the geometrical factors for the nitrile group represented by the magenta star were varied in the calculation. 


\section{Dynamics of $\mathrm{Li}^{+}$solvation shell}

The dynamics of the ACN molecules in the $\mathrm{Li}(\mathrm{ACN})_{4}{ }^{+}$complex can be retrieved from the waiting time evolution of the coordinated peak shapes in the 2DIR spectra and represents the dynamics of the frequency-frequency correlation function (FFCF). In any tetrahedral solvation shell, the Hamiltonian of the $\mathrm{CN}$ stretch can be described as

$$
\begin{aligned}
H(t) & =\left[\begin{array}{cccc}
\omega_{10}+\delta \omega_{1}(t) & \beta_{12}(t) & \beta_{13}(t) & \beta_{14}(t) \\
\beta_{12}(t) & \omega_{10}+\delta \omega_{2}(t) & \beta_{23}(t) & \beta_{24}(t) \\
\beta_{13}(t) & \beta_{23}(t) & \omega_{10}+\delta \omega_{3}(t) & \beta_{34}(t) \\
\beta_{14}(t) & \beta_{24}(t) & \beta_{34}(t) & \omega_{10}+\delta \omega_{4}(t)
\end{array}\right] \\
& =\left[\begin{array}{cccc}
\omega_{10}^{(1)}(t) & 0 & 0 & 0 \\
0 & \omega_{10}^{(2)}(t) & 0 & 0 \\
0 & 0 & \omega_{10}^{(3)}(t) & 0 \\
0 & 0 & 0 & \omega_{10}^{(4)}(t)
\end{array}\right],
\end{aligned}
$$

where $\omega_{10}$ is the transition frequency for each isolated $\mathrm{CN}$ stretch, $\delta \omega_{i}(t)$ describes the time-dependent change in frequency for the $\mathrm{i}$-th $\mathrm{CN}$ stretch, and $\beta_{\mathrm{ij}}(\mathrm{t})$ defines the time-dependent coupling between the $i$-th and $j$-th $\mathrm{CN}$ stretches. Using the previously demonstrated assumption that $\beta_{\mathrm{ij}}(\mathrm{t})$ is negligible, the Hamiltonian reduces to a simple expression containing four degenerate frequencies $\left(\omega_{10}^{(j)}(t)=\omega_{10}+\delta \omega_{j}(t)\right)$, where each randomly fluctuates according to its $\delta \omega_{\mathrm{j}}(\mathrm{t})$. A similar Hamiltonian is deduced for the CIP but only contains three sites. Thus, the characteristic time of FFCF directly relates to the fluctuation of the individual sites.

The spectral diffusion of ACN molecules coordinated to $\mathrm{Li}^{+}$, computed using the center line slope (CLS), ${ }^{81,82}$ is shown in Fig. 6. The CLSs of the coordinated CN stretches are well modeled with a single exponential decay of the form: $f\left(T_{w}\right)=A * e^{\left(-T_{w} / \tau\right)}+y_{0}$, where $T_{w}$ is the waiting time, $A$ is the amplitude, $\tau$ is the decorrelation time, and $y_{0}$ is the offset. The model parameters (Table I) reveal that the ACN molecules undergo thermal frequency fluctuations in time scales smaller than 2 ps. Moreover, the presence of an offset $\left(\mathrm{y}_{0}\right)$ is also observed, which is indicative of the 2DIR peak containing more than one underlying transition that exchanges with a slow characteristic time. For example, the CLS offset in the LiTFSI sample could be attributed to the presence of multiple transitions and/or solvent separated ion pairs. While the former case refers to the presence of the four/three $\mathrm{CN}$ stretches within the same band,

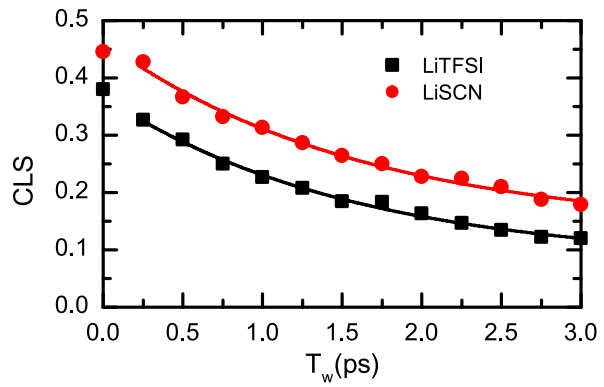

FIG. 6. CLS of coordinated ACN in LiTFSI and LiSCN solutions with $X\left(\mathrm{Li}^{+}\right)=0.05$. Black and red represent the data of LiTFSI and LiSCN, respectively. The lines correspond to the model fitting as described in the text.
TABLE I. Model parameters from the fitting of coordinated CLS in LiTFSI and LiSCN in ACN solutions.

\begin{tabular}{lccc}
\hline \hline Sample & $\tau(\mathrm{ps})$ & $\mathrm{y}_{0}$ & $\mathrm{~A}$ \\
\hline ACN/LiTFSI & $1.6 \pm 0.2$ & $0.08 \pm 0.01$ & $0.29 \pm 0.01$ \\
ACN/LiSCN & $1.6 \pm 0.2$ & $0.13 \pm 0.02$ & $0.33 \pm 0.01$ \\
\hline
\end{tabular}

the latter case produces an offset since it is expected that the making and breaking of solvent separated ion pairs occur with a much longer characteristic time. ${ }^{52}$ A curious finding is that the CLS of the coordinated $\mathrm{CN}$ stretch has a very similar value of the spectral diffusion time ( $\tau=1.6 \mathrm{ps}$ ) for both samples even though $\mathrm{Li}^{+}$form totally different solvation shells corresponding to a free ion and CIP, as previously described. In other words, the motions of the ACN molecules coordinating $\mathrm{Li}^{+}$appear to be very similar even in the presence of an anion in the $\mathrm{Li}^{+}$solvation shell. It should be noted that the CLS dynamics of the coordinated ACN molecules only accounts for site frequency fluctuation and not their reorientations ${ }^{84}$ since the $\mathrm{Li}(\mathrm{ACN})_{4}{ }^{+}$complex has a large molecular size $\left(148 \mathrm{~cm}^{3} / \mathrm{mol}\right)$ compared to a single ACN molecule $\left(38 \mathrm{~cm}^{3} / \mathrm{mol}\right)$. This is in agreement with the reorientation dynamics of 33 ps and 24 ps for a single coordinated ACN (see the supplementary material) computed from the AIMDSs for $\mathrm{Li}(\mathrm{ACN})_{4}{ }^{+}$and $\mathrm{Li}(\mathrm{ACN})_{3}(\mathrm{SCN})$, respectively. Thus, the fast dynamics seen in CLS of the coordinated peak describes exclusively the thermal motions of the ACN molecules coordinated to $\mathrm{Li}^{+}$.

\section{Molecular mechanism of FFCF}

The molecular mechanism giving rise to the FFCF dynamics was studied using the AIMDSs. Given the simplicity of the $\mathrm{Li}^{+}$solvation shells for the free ion and CIP, it is possible to model the effect of the solvation shell with three structural parameters: the $\mathrm{N} \cdots \mathrm{Li}^{+}$distance, the $\mathrm{N} \cdots \mathrm{Li}^{+} \cdots \mathrm{N}$ angle, and the $\mathrm{C} \equiv \mathrm{N} \cdots \mathrm{Li}^{+}$angle. In the case of $\mathrm{ACN}$, each coordinated $\mathrm{ACN}$ molecule in the $\mathrm{Li}(\mathrm{ACN})_{4}{ }^{+}$complex undergoes fluctuations of its $\mathrm{CN}$ stretch independently of the others due to the weak vibrational coupling between coordinated ACN nitrile stretches. Moreover, the weak coupling makes the frequency of coordinated ACN nitrile stretches to fluctuate slightly when the $\mathrm{N} \cdots \mathrm{Li}^{+} \cdots \mathrm{N}$ angle is changed (Fig. 5). The lack of change in the $\mathrm{CN}$ stretch frequencies with this geometrical factor is in agreement with the single site representation in which changes in the distance and angle between the nitrile group of ACN and $\mathrm{Li}^{+}$drive the large frequency fluctuations in the $\mathrm{CN}$ stretch. Thus, the modeling of the CN stretch coordinated to $\mathrm{Li}^{+}$reduces to two structural factors: the $\mathrm{N} \cdots \mathrm{Li}^{+}$distance and the $\mathrm{C} \equiv \mathrm{N} \cdots \mathrm{Li}^{+}$angle. Interestingly, the autocorrelation function of these two geometrical factors reveals dynamics with characteristic times in the range of 1 ps-2 ps (see the supplementary material), which are both very close to the experimental values of the FFCF. Thus, the dynamics of the structural factors alone does not show to what degree each geometrical factor contributes to the FFCF. To this end, a frequency map of the $\mathrm{CN}$ stretch was built (see the supplementary material).

The new-built frequency map (see the supplementary material) reflects the effect on the $\mathrm{CN}$ stretch frequency of single $\mathrm{ACN}$ in the $\mathrm{Li}(\mathrm{ACN})_{4}{ }^{+}$or $\mathrm{Li}(\mathrm{ACN})_{3}(\mathrm{SCN})$ complex for the distance $\mathrm{dr}$ 
$\left(\mathrm{N} \cdots \mathrm{Li}^{+}\right)$and the angle $\theta\left(\mathrm{C} \equiv \mathrm{N} \cdots \mathrm{Li}^{+}\right)$. It is also observed that the frequency fluctuations due to changes in the angle $\theta$ are not significantly affected by the changes in the distance $\mathrm{dr}$ and vice versa (Fig. 5). This indicates that frequency changes can be described in the first approximation by two independent fluctuation terms as

$$
\omega_{01}(t)=\omega_{01}^{o p t}+\delta \omega_{\theta}(t)+\delta \omega_{r}(t)
$$

where $\delta \omega_{\theta}$ and $\delta \omega_{r}$ are the fluctuations of the frequency due to changes in the angle $\theta$ and distance $\mathrm{dr}$, respectively. Using the DFT map and the coordinates from the AIMDSs, the instantaneous frequencies were calculated for $\mathrm{ACN}$ in the $\mathrm{Li}^{+}$solvation of the ACN/LiTFSI and ACN/LiSCN systems.

The theoretical FFCF derived from the instantaneous frequencies shows an ultrafast decay in agreement with the fast fluctuations seen for the angle $\theta$ and distance dr (Fig. 7). The theoretical FFCF modeled with a single exponential decay (Table II) has characteristic times of 1 ps-2 ps for the $\mathrm{CN}$ stretches in $\mathrm{Li}(\mathrm{ACN})_{4}{ }^{+}$and $\mathrm{Li}(\mathrm{ACN})_{3}(\mathrm{SCN})$. These time scales are in good agreement with their experimental counterpart of $1.6 \pm 0.2$ ps. In addition, the computed FFCF reveals the presence of another ultrafast component ( $\sim 0.1 \mathrm{ps}$ ), but it is likely that this component only contributes as a homogeneous component in the FFCF, and it is not measured experimentally. ${ }^{82,85}$ To compute the influence of the $\mathrm{dr}$ and $\theta$ geometrical factors on the FFCF, the frequency autocorrelation function was computed for each individual geometrical factors (angle $\theta$ and distance dr). The FFCFs for the individual components (Fig. 7) reveal the same decorrelation times of $1 \mathrm{ps}-2 \mathrm{ps}$. However, it is evident that for both the ACN/LiTFSI and ACN/LiSCN systems, the frequency of a single ACN nitrile stretch is dominated by the fluctuations in the angle $\theta$ since the fluctuations of distance $\mathrm{dr}$ have a much smaller amplitude (variance) as compared to those of the angle $\theta$ or to the

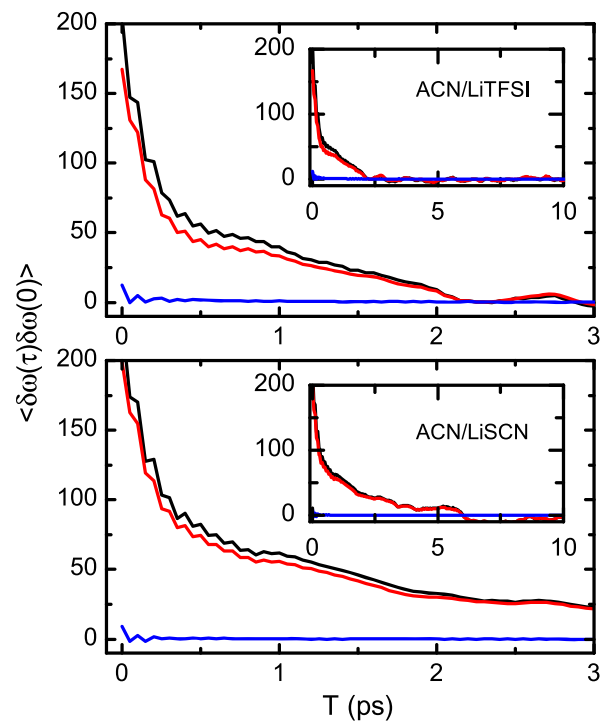

FIG. 7. FFCFs computed from the AIMDS for the ACN/LiTFSI (top panel) and ACN/LiSCN (bottom panel) systems. Insets show the FFCFs for 10 ps windows. Black, red, and blue lines represent the FFCF containing both dr and $\theta$, only $\theta$, or only dr, respectively.
TABLE II. Parameters from fitting the autocorrelation functions of both $\mathrm{dr}$ and $\theta$, only $\theta$, or only dr in ACN/LiTFSI and ACN/LiSCN systems.

\begin{tabular}{lccc}
\hline \hline Type & Fitting parameters & ACN/LiTFSI & ACN/LiSCN \\
\hline \multirow{2}{*}{ Both dr and $\theta$} & $A^{\prime}$ & $104 \pm 4$ & $109 \pm 2$ \\
& $\tau^{\prime}(\mathrm{ps})$ & $0.88 \pm 0.03$ & $1.70 \pm 0.04$ \\
Only $\theta$ & $A^{\prime}$ & $82 \pm 3$ & $99 \pm 2$ \\
& $\tau^{\prime}(\mathrm{ps})$ & $0.92 \pm 0.04$ & $1.72 \pm 0.04$ \\
Only dr & $A^{\prime}$ & $1.9 \pm 0.1$ & $0.65 \pm 0.05$ \\
& $\tau^{\prime}(\mathrm{ps})$ & $1.44 \pm 0.08$ & $0.94 \pm 0.08$ \\
\hline
\end{tabular}

total amplitude of the frequency fluctuation (Fig. 7). The result is also in line with the effect of individual motions of ACN molecules on the solvation shell of $\mathrm{Li}^{+}$, where only the angle $\theta$ has a strong effect on the frequency of the ACN nitrile stretch (Fig. 5). In addition, the strong interaction between $\mathrm{Li}^{+}$and the $\mathrm{CN}$ group, as seen by the presence of sharp peaks in the radial distribution functions (Fig. 4), is likely to restrict the fluctuations of the distance dr as compared to the angle $\theta$, which explains the small effect of $\mathrm{dr}$ on the FFCF. Overall, the results demonstrate that angles play a significant role in the frequency fluctuations of coordinated nitriles and are similar to what was previously reported in carbonate-based electrolytes, where it was postulated that the angles within the first solvation shell of $\mathrm{Li}^{+}$are key for describing the FFCF of the coordinated carbonyl groups. ${ }^{12,14}$

\section{SUMMARY}

The present study investigated the dynamics of the acetonitrile molecules in the first solvation shell of $\mathrm{Li}^{+}$and unveiled the underlying molecular mechanism of such dynamics by a combination of experimental and theoretical methodologies. The results showed that $\mathrm{Li}(\mathrm{ACN})_{4}{ }^{+}$and $\mathrm{Li}(\mathrm{ACN})_{3}(\mathrm{SCN})$ are formed in ACN/LiTFSI and ACN/LiSCN systems, respectively. In addition, it is observed that the ACN molecules solvating $\mathrm{Li}^{+}$are vibrationally weakly coupled. Thus, FFCF of the coordinated CN stretch extracted from 2DIR experiments and the AIMDSs revealed that the in-place motions of the individual ACN molecules in the $\mathrm{Li}^{+}$first solvation shell have a characteristic time of $\sim 1.6$ ps irrespective of the speciation of $\mathrm{Li}^{+}$. The experimental FFCF was found to be in good agreement with the theoretical FFCF derived from the AIMDSs and the DFT frequency maps. In addition, an analysis of the effect of the two geometrical factors, the distance $\mathrm{dr}$ and the angle $\theta$, on FFCF indicated that the angle $\theta$ is the dominating factor of the FFCF dynamics, while the contribution of the distance $\mathrm{dr}$ is found to be negligible. The findings of this work are in line with the previous modeling of the solvation shell motions of $\mathrm{Li}^{+}$.

\section{SUPPLEMENTARY MATERIAL}

See the supplementary material for dielectric constants and transition dipole moment magnitudes of $\mathrm{ACN}, \mathrm{BC}$, and $\mathrm{DMC}$ (Table S1), calculated energy results of $\mathrm{Li}^{+}$binding $\mathrm{SCN}^{-}$through $\mathrm{S}$ or $\mathrm{N}$ (Table S2), modeling of reorientation dynamics of coordinated ACN in LiTFSI and LiSCN solutions (Table S3), modeling of correlation functions of dr or angle $\theta$ in LiTFSI and LiSCN systems 
(Table S4), FTIR spectra of Li salts in ACN-d3 in the thiocyanate $\mathrm{CN}$ stretching region (Fig. S1), 2DIR spectra of 0.05 LiTFSI or 0.05 LiSCN in ACN-d3 at waiting time 0 (Fig. S2), g(r) and integrated $\mathrm{g}(\mathrm{r})$ between $\mathrm{Li}^{+}$and $\mathrm{N}$ of SCN${ }^{-}$(Fig. S3), anisotropy of coordinated ACN in LiTFSI and LiSCN solutions with time (Fig. S4), correlation functions of $\theta$ or $\mathrm{dr}$ of coordinated $\mathrm{CN}$ of ACN (Fig. S5), FFCFs computed from the AIMDS for LiTFSI and LiSCN systems (Fig. S6), dr ( $\mathrm{Li}-\mathrm{N})$ and $\theta(\mathrm{Li}-\mathrm{N}-\mathrm{C})$ distribution of LiTFSI and LiSCN electrolytes from the AIMD simulation (Fig. S7), frequency fluctuations due to the change in $\theta$ (left) or $\mathrm{dr}$ (right) in Li solvation shells (Fig. S8), the frequency map of coordinated ACN built from DFT computations of solvated first solvation shells (Fig. S9), the snapshot of optimized $\mathrm{Li}(\mathrm{ACN})_{4}{ }^{+}$(Fig. S10), the snapshot of optimized $\mathrm{Li}(\mathrm{ACN})_{3} \mathrm{SCN}$ (Fig. S11), the snapshot of optimized solvated $\mathrm{Li}(\mathrm{ACN})_{4}{ }^{+}$(Fig. S12), and the snapshot of optimized solvated $\mathrm{Li}(\mathrm{ACN})_{3} \mathrm{SCN}$ (Fig. S13).

\section{ACKNOWLEDGMENTS}

The authors would like to acknowledge financial support from the National Science Foundation (Grant Nos. CHE-1751735). The authors would also like to acknowledge the computer time provided by the High Performance Computing Center at Louisiana State University and the Louisiana Optical Network Initiative (LONI).

\section{DATA AVAILABILITY}

The data that support the findings of this study are available from the corresponding author upon reasonable request.

\section{REFERENCES}

${ }^{1}$ K. Xu, Chem. Rev. 104, 4303 (2004).

${ }^{2}$ K. Xu, Chem. Rev. 114, 11503 (2014).

${ }^{3}$ E. Peled and S. Menkin, J. Electrochem. Soc. 164, A1703 (2017).

${ }^{4}$ M. Marcinek et al., Solid State Ionics 276, 107 (2015).

${ }^{5}$ A. M. Chockla et al., J. Am. Chem. Soc. 133, 20914 (2011).

${ }^{6}$ P. L. Taberna et al., Nat. Mater. 5, 567 (2006).

${ }^{7}$ Y.-Y. Hu et al., Nat. Mater. 12, 1130 (2013).

${ }^{8}$ A. M. Haregewoin, A. S. Wotango, and B.-J. Hwang, Energy Environ. Sci. 9, 1955 (2016).

${ }^{9}$ A. L. Michan et al., Chem. Mater. 28, 8149 (2016).

${ }^{10}$ D. M. Seo et al., J. Phys. Chem. C 119, 14038 (2015).

${ }^{11}$ K. D. Fulfer and D. G. Kuroda, J. Phys. Chem. C 120, 24011 (2016).

${ }^{12}$ K. D. Fulfer and D. G. Kuroda, Phys. Chem. Chem. Phys. 19, 25140 (2017).

${ }^{13}$ K. D. Fulfer and D. G. Kuroda, Phys. Chem. Chem. Phys. 20, 22710 (2018).

${ }^{14}$ X. Zhang and D. G. Kuroda, J. Chem. Phys. 150, 184501 (2019).

${ }^{15}$ K.-K. Lee et al., Nat. Commun. 8, 14658 (2017).

${ }^{16}$ J. Lim et al., J. Phys. Chem. B 123, 6651 (2019).

${ }^{17}$ C. Liang, K. Kwak, and M. Cho, J. Phys. Chem. Lett. 8, 5779 (2017).

${ }^{18}$ J. Wang et al., Nat. Commun. 7, 12032 (2016).

${ }^{19}$ Y. Umebayashi et al., J. Phys. Chem. B 111, 13028 (2007).

${ }^{20}$ Y. Yamada et al., J. Am. Chem. Soc. 136, 5039 (2014).

${ }^{21}$ L. Suo et al., Science 350, 938 (2015).

${ }^{22}$ J.-C. Soetens et al., J. Mol. Liq. 92, 201 (2001).

${ }^{23}$ K. Fujii et al., J. Phys. Chem. C 121, 22720 (2017).

${ }^{24}$ L. Yang, A. Xiao, and B. L. Lucht, J. Mol. Liq. 154, 131 (2010).

${ }^{25}$ X. Bogle et al., J. Phys. Chem. Lett. 4, 1664 (2013).

${ }^{26}$ K. Hayamizu, J. Chem. Eng. Data 57, 2012 (2012).

${ }^{27}$ Y. Matsuda et al., J. Electrochem. Soc. 149, A1045 (2002).
${ }^{28}$ A. Cresce and K. Xu, Electrochem. Solid-State Lett. 14, A154 (2011).

${ }^{29}$ T. Fukushima et al., Electrochem. Solid-State Lett. 4, A127 (2001).

${ }^{30}$ N. H. C. Lewis et al., J. Phys. Chem. C 124, 3470 (2020).

${ }^{31}$ X. Chen et al., J. Phys. Chem. B 123, 9889 (2019).

${ }^{32}$ J. C. Rushing, F. M. Leonik, and D. G. Kuroda, J. Phys. Chem. C 123, 25102 (2019).

${ }^{33}$ L. Bao et al., Sci. Rep. 7, 45921 (2017).

${ }^{34}$ O. Borodin et al., J. Phys. Chem. C 117, 7433 (2013).

${ }^{35}$ T. P. Pollard and T. L. Beck, J. Chem. Phys. 147, 161710 (2017).

${ }^{36}$ O. Borodin et al., Phys. Chem. Chem. Phys. 18, 164 (2016).

${ }^{37}$ N. Kumar and J. M. Seminario, J. Phys. Chem. C 120, 16322 (2016).

${ }^{38}$ J. Barthel, R. Buchner, and E. Wismeth, J. Solution Chem. 29, 937 (2000).

${ }^{39}$ M. D. Bhatt, M. Cho, and K. Cho, Appl. Surf. Sci. 257, 1463 (2010).

${ }^{40}$ T.-M. Chang and L. X. Dang, J. Chem. Phys. 147, 161709 (2017).

${ }^{41}$ P. Hamm and M. Zanni, Concepts and Methods of 2D Infrared Spectroscopy (Cambridge University Press, Cambridge, 2011).

${ }^{42}$ W. R. Fawcett et al., J. Chem. Soc., Faraday Trans. 89, 811 (1993).

${ }^{43}$ A. K. Mollner et al., J. Phys. Chem. A 108, 3344 (2004).

${ }^{44}$ X. Xuan et al., J. Phys. Chem. A 108, 7513 (2004).

${ }^{45}$ W. R. Fawcett, G. Liu, and T. E. Kessler, J. Phys. Chem. 97, 9293 (1993).

${ }^{46}$ O. Borodin and G. D. Smith, J. Phys. Chem. B 110, 4971 (2006).

${ }^{47}$ D. M. Seo et al., J. Electrochem. Soc. 159, A1489 (2012).

${ }^{48}$ A. Webber, J. Electrochem. Soc. 138, 2586 (1991).

${ }^{49}$ D. M. Seo et al., J. Electrochem. Soc. 159, A553 (2012).

${ }^{50}$ M. Ji, S. Park, and K. J. Gaffney, J. Phys. Chem. Lett. 1, 1771 (2010).

${ }^{51}$ D. Paoli, M. Luçon, and M. Chabanel, Spectrochim. Acta, Part A 34, 1087 (1978).

${ }^{\mathbf{5 2}}$ K.-K. Lee et al., J. Chem. Phys. 134, 064506 (2011).

${ }^{53}$ M. C. Asplund, M. T. Zanni, and R. M. Hochstrasser, Proc. Natl. Acad. Sci. U. S. A. 97, 8219 (2000).

${ }^{54}$ A. C. Eckbreth, Appl. Phys. Lett. 32, 421 (1978).

${ }^{55}$ D. G. Kuroda and R. M. Hochstrasser, J. Chem. Phys. 135, 204502 (2011).

${ }^{56}$ K. P. Sokolowsky and M. D. Fayer, J. Phys. Chem. B 117, 15060 (2013).

${ }^{57}$ S. Park, M. Ji, and K. J. Gaffney, J. Phys. Chem. B 114, 6693 (2010).

${ }^{58}$ Y. S. Kim, J. Wang, and R. M. Hochstrasser, J. Phys. Chem. B 109, 7511 (2005).

${ }^{59}$ J. VandeVondele et al., Comput. Phys. Commun. 167, 103 (2005).

${ }^{60}$ S. Grimme, J. Comput. Chem. 27, 1787 (2006).

${ }^{61}$ S. Goedecker, M. Teter, and J. Hutter, Phys. Rev. B: Condens. Matter Mater.

Phys. 54, 1703 (1996).

${ }^{62}$ G. Lippert et al., J. Phys. Chem. 100, 6231 (1996).

${ }^{63}$ J. P. Perdew, K. Burke, and M. Ernzerhof, Phys. Rev. Lett. 77, 3865 (1996).

${ }^{64}$ P. Ray et al., J. Phys. Chem. B 121, 5279 (2017).

${ }^{65}$ K. Leung and J. L. Budzien, Phys. Chem. Chem. Phys. 12, 6583 (2010).

${ }^{66}$ S. Hwang et al., J. Phys. Chem. C 122, 19438 (2018).

${ }^{67}$ M. J. Frisch, G. W. Trucks, H. B. Schlegel, G. E. Scuseria et al., Gaussian 09, 2013.

${ }^{68}$ L. Martínez et al., J. Comput. Chem. 30, 2157 (2009).

${ }^{69}$ J. A. Maier et al., J. Chem. Theory Comput. 11, 3696 (2015).

${ }^{70}$ D. A. Case, R. M. Betz, D. S. Cerutti, T. E. Cheatham III, T. A. Darden, R. E. Duke, T. J. Giese, H. Gohlke et al., AMBER 2016 (University of California, San Francisco, 2016).

${ }^{71}$ I. Suzuki, J. Nakagawa, and T. Fujiyama, Spectrochim. Acta, Part A 33, 689 (1977).

${ }^{72}$ S. Hashimoto, T. Ohba, and S.-i. Ikawa, Chem. Phys. 138, 63 (1989).

${ }^{73}$ D. Jamroz, J. Stangret, and J. Lindgren, J. Am. Chem. Soc. 115, 6165 (1993).

${ }^{74}$ V. A. Sajeevkumar and S. Singh, J. Mol. Struct. 382, 101 (1996).

${ }^{75}$ J.-S. Seo, B.-S. Cheong, and H.-G. Cho, Spectrochim. Acta, Part A 58, 1747 (2002).

${ }^{76}$ L. Liu and H. J. Bakker, Phys. Rev. Lett. 112, 258301 (2014).

${ }^{77}$ K. A. See et al., ACS Appl. Mater. Interfaces 8, 34360 (2016).

${ }^{78}$ D. M. Seo et al., J. Electrochem. Soc. 160, A1061 (2013). 
${ }^{79}$ R. Puchta et al., Inorg. Chem. 43, 8227 (2004).

${ }^{80}$ D. Spångberg and K. Hermansson, Chem. Phys. 300, 165 (2004).

${ }^{81}$ F. Šanda et al., J. Phys. Chem. A 119, 10893 (2015).

${ }^{82}$ E. E. Fenn and M. D. Fayer, J. Chem. Phys. 135, 074502 (2011).
${ }^{83}$ D. G. Kuroda, D. Y. Vorobyev, and R. M. Hochstrasser, J. Chem. Phys. 132, 044501 (2010).

${ }^{84}$ P. L. Kramer et al., J. Chem. Phys. 142, 184505 (2015).

${ }^{85} \mathrm{~K}$. Kwak et al., J. Chem. Phys. 127, 124503 (2007). 